\title{
Gateway sink placement for sensor node grid distribution in LoRa smart city networks
}

\author{
Nur Aishah Bt. Zainal, Mohamed Hadi Habaebi, Israth Jahan Chowdhury, Md Rafiqul Islam, \\ Jamal I. Daoud \\ Electrical and Computer Engineering Department, International Islamic University Malaysia, Malaysia
}

\begin{tabular}{l}
\hline \hline Article Info \\
\hline Article history: \\
Received Sep 30, 2018 \\
Revised Nov 26, 2018 \\
Accepted Dec 8, 2018
\end{tabular}

Keywords:

Energy security

Grid distribution

LoRa

Sink placement

Smart city

\begin{abstract}
Low Power Wide Area Network (LPWAN) is a type of wireless communication network designed to allow long range communications at a low bit rate among things (connected objects), such as sensors operated on a battery. It is a new technology that operates in unauthorized spectrum which designed for wireless data communication [1]. It is used in Internet of Thing (IoT) applications and M2M communications. It provides multi-year battery lifetime and is intended for sensors and applications that need to transmit only a few information over long distances a few times per hour from different environments. In order to have an insight of such long range technology, this paper evaluates the performance of LoRa radio links under shadowing effect and realistic smart city utilities node grid distribution. Such environment is synonymous to residential, industrial and modern urban centers. The focus is to include the effect of shadowing on the radio links while attempting to study the optimum sink node numbers and their locations for maximum sensor node connectivity. Results indicate that the usual unrealistic random node distribution does not reflect actual real-life scenario where many of the these sensing nodes follow the utilities infrastructure around the city (e.g., street light posts, water and gas delivery pipes,...etc). The system is evaluated in terms of connectivity and packet loss ratio.
\end{abstract}

Copyright $@ 2019$ Institute of Advanced Engineering and Science. All rights reserved.

\section{Corresponding Author:}

Mohamed Hadi Habaebi,

Department of Electrical and Computer Engineering,

International Islamic University Malaysia,

Jalan Gobmak, Kuala Lumpur, WP, 53100, Malaysia.

Email: habaebi@iium.edu.my

\section{INTRODUCTION}

In the late 1980s and mid-1990s, there were identical topologies and system structures, yet they were not called LPWANs. They are the forerunners to this development. With the development of science and technologies, people are being exposed to the benefit of using internet. With internet, life getting easier. People can easily get in touch even though they are not in the same place. In addition, they want the service with low power consumption but greater connections. The new wireless technologies have been produced by the name Low Power Wide Area (LPWA), with the special characteristics that suitable for the implementation of IoT applications which also include a simplified network topology, power optimized radio network, frame sizes transmitted several times in a day at ultra-low speeds and upstream communication model that enable the end-devices to stay in low energy mode[2]. With this setting, it enables a range of kilometers with longer battery life; which up to ten years of operation, low cost devices with plain but scalable deployments and thin foundation. The LPWA features, makes it possible for IoT to function well with only use a very low bit-rate of data for reporting and does not need frequent changes of the batteries because of the low-powered features of the LPWA[2]. 
The topic is concern about the performance of LoRa (Long Range) Radio Link which is one of the LPWA technologies which promising wide area for IoT technologies that was proposed by Semtech company and later being promoted by the LoRa Alliance. LoRa used chirp modulation technology, which allowing for long range transmission with low power and low cost for designing. This can be achieved by using the spread spectrum technique accommodating several devices in a channel. The termed LoRaWan has been defined as the higher layers and system architecture on top the LoRa physical layers. With all these attributes, it makes LoRa suitable to be used for IoT [3].

The objective of this paper is to evaluate the performance of LoRa shadowed radio links, typical in urban and semi urban centers, together with node grid distribution and optimal sink node placement using measures of connectivity and packet loss ratios. The paper is organized as follows. Section 2 introduces a review of the open literature. Section 3 discusses the research methodology. Section 4 presents the results and discussion and the paper id concluded in Section 5.

\section{RESEARCH METHOD}

Microchip Technology Inc., which is outstanding as a main supplier of microcontroller, mixed-signal, analog as well as Flash-IP solutions, reported the first in a progression of modules for the LoRa remote systems administration standard. It empowers Internet of Things (IoT) and Machine-to-Machine (M2M) correspondence with a range more than 10miles (for rural condition), longer battery life which about more noteworthy than 10 years, and the ability to associate a great many remote sensor hubs to LoRaWAN gateways. [4] LoRa (Long Range) is a modulation technique the gives a notable longer range. It is based on spread-spectrum techniques and differences of the chirp spread spectrum (CSS) and incorporated with Forward Error Correction (FEC). LoRa utilizes whole channel data transfer capacity to transmit the data, which make it strong to channel noise and insensitive to frequency offsets. Likewise, it can demodulate signals $19.5 \mathrm{~dB}$ underneath the noise floor, while most regular Frequency Shift Keying (FSK) frameworks require a signal energy of between $8 \mathrm{~dB}$ to $10 \mathrm{~dB}$ over the noise floor in order to demodulate effectively. This modulation is the physical layer which, it can be utilized by different protocol architectures. [5]

LoRaWAN is a Medium Access Control (MAC) protocol for a high capacity that the LoRa Alliance is standardized for Low Power Wide Area Network (LPWAN). This protocol is optimized for low cost battery worked sensors and incorporates distinctive classes of nodes to optimize the trade-off between battery life and network latency. The architecture of this protocol was expected to effortlessly find and track portable objects for asset tracking. It is one of the quickest developing applications for the Internet of Things (IoT). The LoRa gateways utilized long range star network topology and being used in a LoRaWAN framework (see Figure 1). Due to the of properties of LoRa, the framework are multi-modem handsets, multi-channel and ready to demodulate on different channels simultaneously and demodulate various signals on the indistinguishable channel in the meantime. The endpoint utilizes distinctive radio frequency with the getaways to permit high limit and execute as a straightforward extension handing-off messages between the end-gadgets and a central system server. End-gadgets utilize a hop remote correspondence to the portals while gateways associated with the system server through standard Internet Protocol (IP) associations. The portal has numerous adaptations and it is relying upon the use limit and coveted establishment area (e.g.: tower versus home)[5].

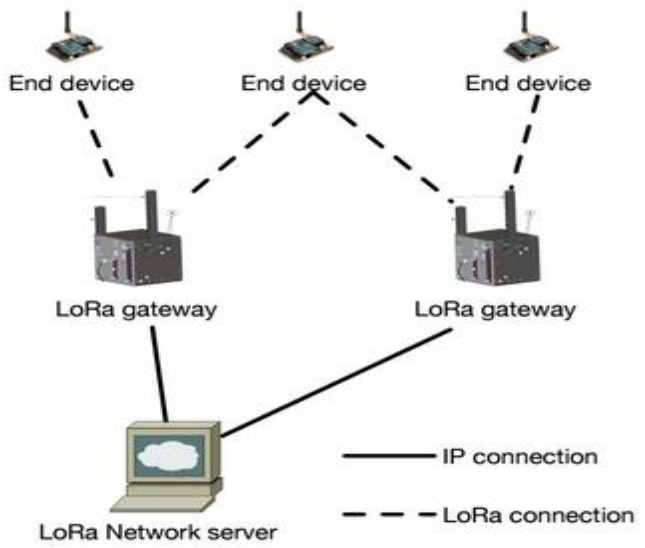

Figure 1. The architecture of LoRa which include three dissimilar devices [6] 
1) LoRaWan Air Interface

LoRa Alliance has released the air interface specific document, which are

a) LoRaWAN air interface v1.0.0

b) LoRaWAN air interface v1.0.1

c) LoRaWAN air interface v1.1 (to be announced soon)

With the development of LoRaWAN air interface specification, the level of security and privacy has guaranteed. The application provider is solely responsible for the encryption of the entire application payload using the AES128 Application Session Key. With this feature, it confirms the security of the payload. Next, 32bit signature has been added which result in computed the entire frame by using a Network session key. This attribute guarantees the originality of the devices and the frame cannot easily modify [7].

The security on the air does not assure whether the network server can easily be hacked or the device is anti-temper. In order to get an anti-temper device, a hardware secure element must use to store and derive the keys. In addition, to implement the cryptographic functions in the network server, it is advisable to use a Hardware Key Management system [7].

2) Long Range and Shadowing Effect

The LoRaWAN communication is expected to cover longer distances; hence, frames can be lost due to propagation loses, and some physical phenomenon such as shadowing effect, reflection, and scattering. All of this need to be taken into account whenever we do the simulation because there is no "perfect" things in real life. Shadowing is the effect that the received signal power varies due to objects blocking the propagation path between transmitter and receiver. These variations are experience on local-mean powers, that is, short-term averages to remove variations due to multipath fading. The shadowing effect is needed in our calculation so that we can emulate real-life system performance and we can predict what will happen in the future and find a solution for it.

Since wide coverage is one of the crucial requirements for the LPWAN networks, Petäjäjärvi et.al (2015) conducted a study using real-life measurements to see the coverage of the LoRa technology [8]. Them taking the measurements using the LoRaMote and Kerlink's LoRa IoT as the base station. The nodes were placed in two different locations which are on the roof-rack of a car (for the mobile node that moving over the ground) and at the radio mast of a boat (for the mobile node that placed on the sea). The base station was placed on the antenna tower on the roof of the University of Oulu at the height $24 \mathrm{~m}$ over the sea level.

The LoRa node was configured to pass on the maximum signal spreading factor to obtain the maximum range. The Transmit Power (TP) was set to $14 \mathrm{dBm}(25 \mathrm{~mW})$. The result shows that, the packet received from a node that placed at sea are higher than node placed at the ground. As the range increase up to $10-15 \mathrm{~km}$, for the mobile node placed on the ground, the packet lost ratio is $74 \%$, while, for the mobile node placed on the sea, as the range increase up to $15-30 \mathrm{~km}$, the packet lost ratio is $38 \%$. This is due to line of sight blockade by some obstacles and interference from other radio systems. The placement of base station needs to be studied thoroughly to identify the exact point that will give the greater throughput.

In another study [9], a long range experiment was conducted to evaluate the result of outdoor range test estimation for LPWA. The result shows that one of the LPWA base station placed at $470 \mathrm{~m}$ above sea level on Three Rock Mountain in Dublin can potentially serve a coverage area of 1380 square kms within an entire estimated area of over 3,800 square kms. A successful real world range evaluation of $25 \mathrm{~km}$ test link operation between a $14 \mathrm{dBm}$ LPWA client test and a base station provides outcome representing a received SNR consistently exceeding $20 \mathrm{~dB}$ over this test link distance. Again, the coverage over water is higher in contrast with the coverage over the ground. The outcome shows that SNR exceeds $20 \mathrm{~dB}$, which is an excellent performance as generally, a signal with an SNR value of $20 \mathrm{~dB}$ or more is recommended for data networks.

However, in both studies placement of sensor nodes was at random rather than systematically patterned. This placement maybe suitable for indoor sensor distribution but not fit to represent the city center utility companies' infrastructure such as: electricity lamb posts, water and gas delivery pipe lines, etc. Hence, our work looks at the systematic grid distribution of these outdoor nodes and the dilemma of finding a sufficient number of sink nodes (gateways) to provide connectivity.

\section{METHODOLOGY}

In this section, the design of the LoRa sensor simulator will be discussed in detailed and the comparison of the changes that had been made with the existing model will be highlighted. The existing simulator that we used was created by Bor et. al [9]. Other simulators were reported in [12], [14]. At first, we had made a several changes to the simulator. To get the real life situation result, we follow the propagation model deduced from measurements in [8] due to its realistic representation of real-life application scenario in a modern urban center, as shown in Table 1.

Indonesian J Elec Eng \& Comp Sci, Vol. 14, No. 2, May 2019 : $834-842$ 
Table 1. Propagation Models Characterisitcs

\begin{tabular}{cccc}
\hline Ref. No. & Path loss exponent $(\mathrm{n})$ & Shadow fading, $\sigma \mathrm{SF}$ & Mean path loss, Lpl(d0) \\
\hline$[8]$ & 2.32 & $7.8 \mathrm{~dB}$ & 128.95 \\
{$[9]$} & 2.08 & - & 127.41 \\
\hline
\end{tabular}

The LoRa purpose built simulation tool captures the specific LoRa link behavior and enables evaluation of large-scale LoRa networks. The changes will be discussed below in Table 2. For this study, we want to identify the number of required sink node gateways to provide connectivity for a grid sensor node distribution in a typical residential city area. Furthermore, we would like to investigate the effect of shadowing on the links while doing so to make our simulation more realistic. Hence, the performance metric would be the percentages of lost packets and connected nodes in the network. Furthermore, we have followed the four experiments done in [8] by changing the setting parameters for the air interface according to Table 3 below. Nodes are placed equally-distant around the sink such that all nodes can reach the sink with the prearranged setting $\mathrm{SN}$ if shadowing effect is not included. The three transmitter configurations SN1, SN2 and SN3 are given in Table 3.

In all settings, it is being imagined that a 20-byte packet is being sent by each node every $16.7 \mathrm{~min}$ representing a realistic application. The chosen setting for SN1 is the most robust LoRa transmitter settings which leads to transmissions with the longest possible airtime of $1712.13 \mathrm{~ms}$, with SN2; the transmission setting leads to the shortest airtime of $7.07 \mathrm{~ms}$ and with SN3 the chosen setting is the one which is used by common LoRaWAN deployments [8].

In order to evaluate the instantaneous as well as the cumulative effects of shadowing on the air interface, several changes were made to the simulator. A credible measurement campaign was conducted in [8] in a modern cosmopolitan urban environment. Hence, their settings were implemented in the simulator. The table below shows the alterations made to the simulator.

Table 2. Changes of Some Ffeatures of the Simulator

\begin{tabular}{|c|c|c|c|}
\hline No. & Existing simulation (by paper [9]) & Alteration & Justification \\
\hline 1. & $\begin{array}{l}\text { Number of Nodes is inputted by the } \\
\text { user }\end{array}$ & $\begin{array}{l}\text { A new code was added to control number of } \\
\text { Nodes where max. number of nodes is set to } \\
140 .\end{array}$ & $\begin{array}{l}\text { For experimental purpose as } \\
\text { the system cannot support } \\
\text { higher number users as results } \\
\text { indicate. }\end{array}$ \\
\hline 2. & $\begin{array}{l}\text { This paper has set the minimum } \\
\text { sensitivity according to the table in ref. } \\
\text { [9]. }\end{array}$ & $\begin{array}{l}\text { The sensitivity levels were modified } \\
\text { according the model in [8]. }\end{array}$ & $\begin{array}{l}\text { Referring to reference [8], the } \\
\text { experiments was conducted in a } \\
\text { modern cosmopolitan urban } \\
\text { environment representing a } \\
\text { typical city setting. }\end{array}$ \\
\hline 3. & $\begin{array}{l}\text { Path loss component was considered } \\
\text { only. No shadowing effect was studied. }\end{array}$ & $\begin{array}{l}\text { The shadowing effect code was added to the } \\
\text { simulator with input parameters derived } \\
\text { from [8]. }\end{array}$ & $\begin{array}{l}\text { Adding the shadowing effect } \\
\text { will give the realistic output } \\
\text { with regards to the } \\
\text { environment. }\end{array}$ \\
\hline 4. & $\begin{array}{l}\text { The base station (or sink) coverage } \\
\text { radius (distance) was very small and } \\
\text { nodes were scattered randomly. }\end{array}$ & $\begin{array}{l}\text { The node distribution code was changed to } \\
\text { accommodate a new grid distribution of } \\
\text { straight line roads representing typical } \\
\text { utilities distribution in residential area with } \\
\text { 50m separation distance. }\end{array}$ & $\begin{array}{l}\text { Atypical utilities distribution } \\
\text { such as lamp-posts, gaz lines, } \\
\text { and water pipes uses similar } \\
\text { grid distribution rather than } \\
\text { random distribution especially } \\
\text { in a city environment. }\end{array}$ \\
\hline 5. & $\begin{array}{l}\text { The axes set was small following the } \\
\text { coverage radius. }\end{array}$ & $\begin{array}{l}\text { The distance coverage is changed to } 2 \mathrm{~km} \text { for } \\
\mathrm{x} \text {-axis and } \mathrm{y} \text {-axis to accommodate the new } \\
\text { propagation model. }\end{array}$ & $\begin{array}{l}\text { Since LoRa technology } \\
\text { promised to cover a wide area, } \\
\text { hence, we increase the distance, } \\
\text { so it implies to the objective. }\end{array}$ \\
\hline
\end{tabular}

To describe the path loss model in a built up area, the log-distance shadowing path loss model was chosen because it is commonly used in free space area. It matches with LoRa environment where LoRa technology claimed to be used in built-up urban areas, free space and larger suburban areas. Below is the logdistance path loss shadowing equation:

$$
\operatorname{Lpl}(\mathrm{d})=\operatorname{Lpl}(\mathrm{d} 0)+10 \mathrm{nlog}(\mathrm{d} / \mathrm{d} 0)+\sigma \mathrm{SF}
$$

where, $\mathrm{Lpl}(\mathrm{d})$ is the path loss in $\mathrm{dB}, \mathrm{Lpl}(\mathrm{d} 0)$ is the mean path loss at the reference distance $\mathrm{d} 0, \mathrm{n}$ is the path loss exponent, $\sigma \mathrm{SF} \sim \mathrm{N}(0 ; \sigma 2)$ is the normal distribution with zero mean, $\sigma 2$ is the variance to account for shadowing effect [8], [10], [11], [13], [15]. 
The system simulation model works as follows. Firstly, seven tests have been conducted using the same setting with differing the number of sinks in every run. The nodes were arranged like a straight line road. The distance between the nodes in a road is $50 \mathrm{~m}$, while the distance between the roads is $135 \mathrm{~m}$. See Figures 5 through 11 for placement of nodes throughout the experiments.

In the first set of experiments we evaluate the standard capacity of LoRa using a simple setup where $\mathrm{N}$ nodes transmit to one sink. In these experiments, standardized transmitter configuration set $\mathrm{SN}=\{\mathrm{TP}, \mathrm{CF}$, SF, BW, CR \} will be used (see Table 4 for definition of air interface parameters).

Table 3. Experiment's Set of Parameters

\begin{tabular}{cccc}
\hline Parameter & SN1 & SN2 & SN3 \\
\hline Spreading Factor & 12 & 6 & 12 \\
Carrier Frequency & 868 & 868 & 868 \\
Transmit Power & 14 & 14 & 14 \\
Bandwidth & 125 & 500 & 125 \\
Coding Rate & $4 / 8$ & $4 / 5$ & $4 / 5$ \\
\hline
\end{tabular}

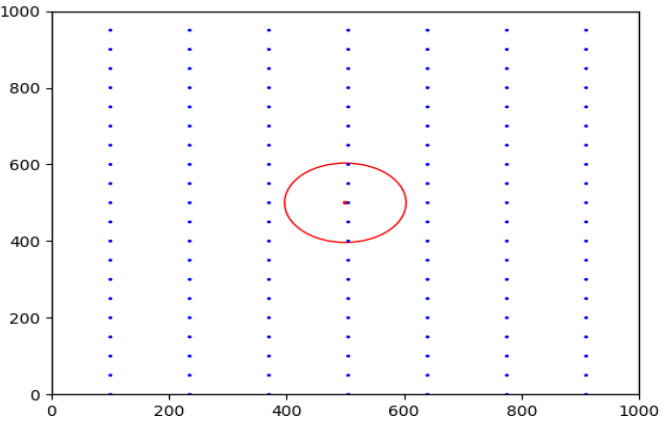

Figure 2. The number of sink is 1 , and the number of nodes are 140

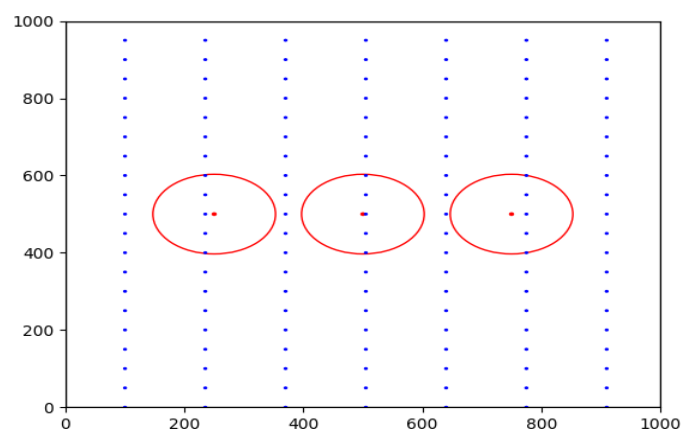

Figure 4. The number of sink is 3 , and the number of nodes are 140

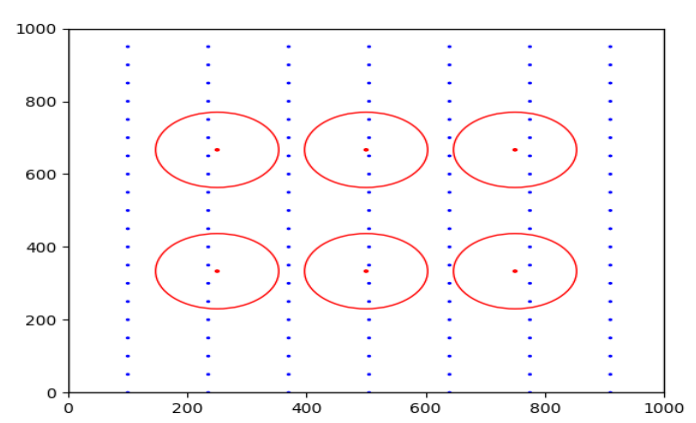

Figure 6. The number of sink is 6 , and the number of nodes are 140

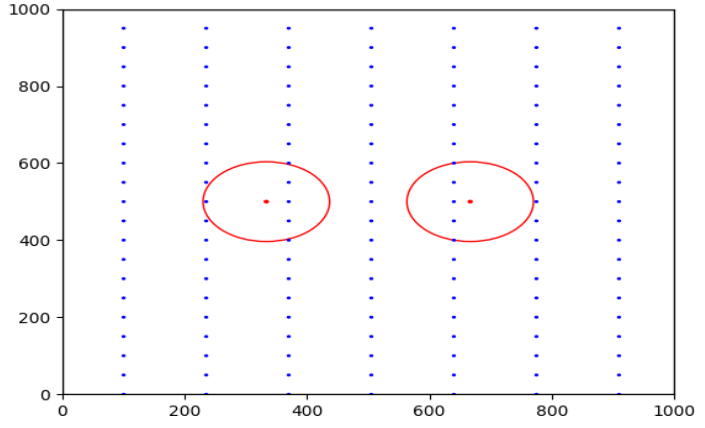

Figure 3. The number of sink is 2 , and the number of nodes are 140

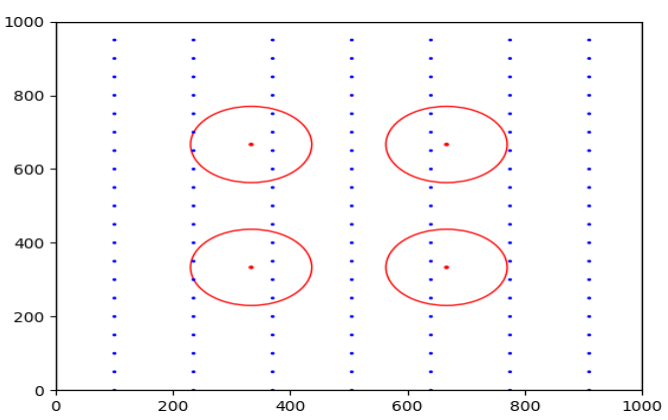

Figure 5. The number of sink is 4 , and the number of nodes are 140

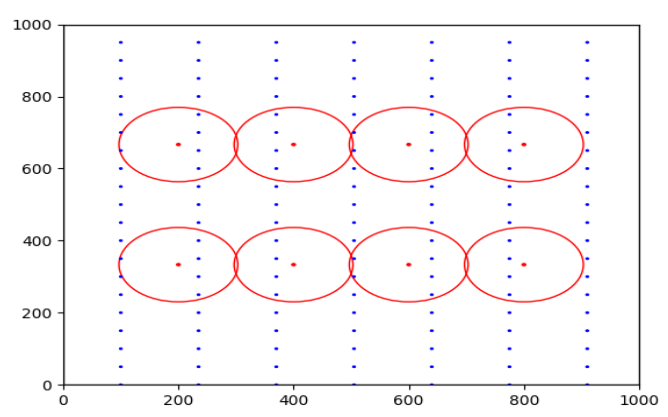

Figure 7. The number of sink is 8 , and the number of nodes are 140 


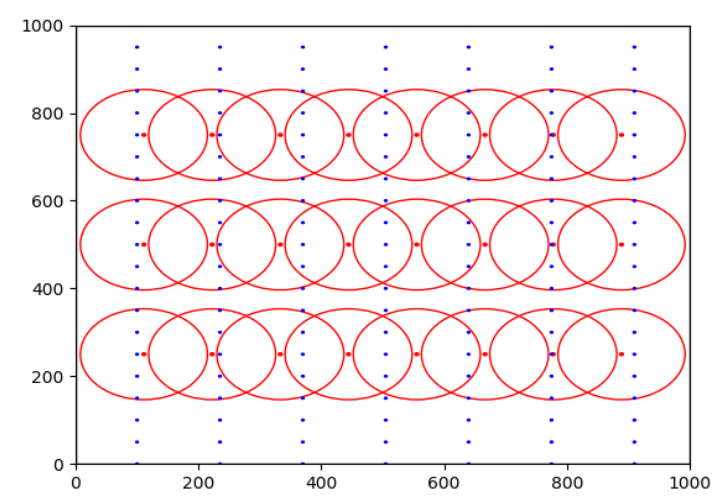

Figure 8 . The number of sink is 24 , and the number of nodes are 140

\section{RESULTS AND DISCUSSION}

The results below show the differences between the updated simulator, SimA (with uniformly girstype distributed nodes around the coverage area) and existing simulator SimB (with randomly distributed nodes), with changes made as stated earlier. The results are consisting of two parts. The first part is the number of sinks against the percentage of covered nodes and number of sinks against covered nodes and uncovered nodes and is presented in Figure 12 and 13(for SimA) and Figure 14 and 15(for SimB), and is summarized in Table 4,5 and 6,7 respectively. The second part is number of sinks against number of lost packet (with five different experiments) and is presented in Figure 16 (for SimA) and 17 (for SimB) and is summarized in Table 8 and 9 respectively.

A. Percentage of Connected (Covered) Nodes $\operatorname{SimA}$

Table 4. Percentage of Covered Nodes with

\begin{tabular}{cc}
\multicolumn{2}{c}{ Various Number of Sinks } \\
\hline No. of Sinks & Covered Nodes (\%) \\
\hline 1 & 3.57 \\
2 & 5.00 \\
3 & 10.71 \\
4 & 12.86 \\
6 & 17.14 \\
8 & 27.14 \\
24 & 62.86 \\
\hline
\end{tabular}

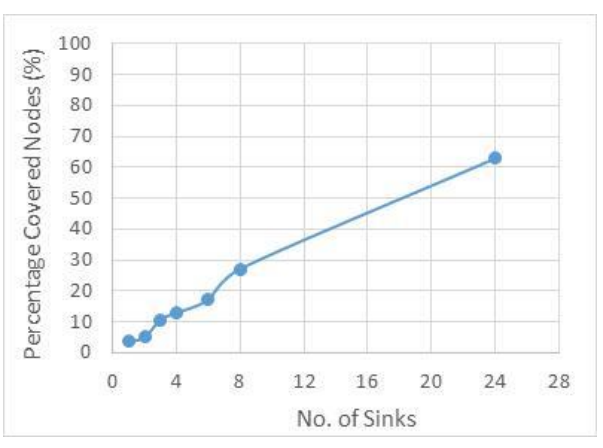

Figure 9. Percentage of covered nodes plotted against number of sinks (Total number of nodes $=140$ )

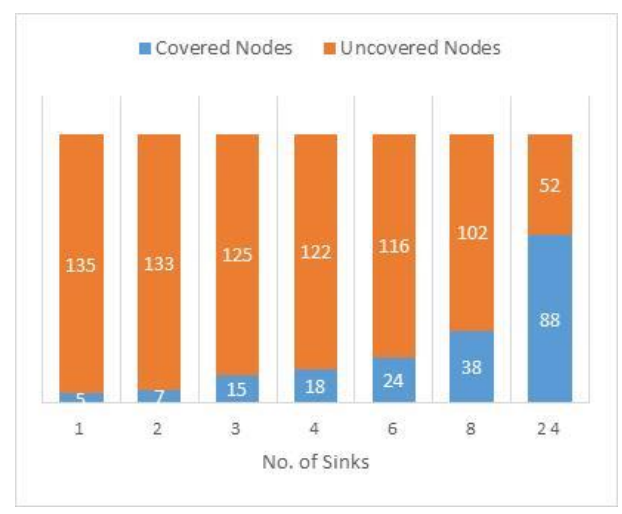

Figure 10. Actual number of nodes covered and uncovered against number of sinks. (Total no of nodes $=140$ )
Table 5. Actual Number of Nodes Covered and Uncovered with Various Number of Sinks Applied (Total number of nodes $=140$ )

\begin{tabular}{ccc}
\hline $\begin{array}{c}\text { No. of } \\
\text { Sinks }\end{array}$ & $\begin{array}{c}\text { Covered } \\
\text { Nodes }\end{array}$ & Uncovered Nodes \\
\hline 1 & 5 & 135 \\
2 & 7 & 133 \\
3 & 15 & 125 \\
4 & 18 & 122 \\
6 & 24 & 116 \\
8 & 38 & 102 \\
24 & 88 & 52 \\
\hline
\end{tabular}


SimB

Table 6. Percentage of Covered Nodes with

\begin{tabular}{cc}
\multicolumn{2}{c}{ Various Number of Sinks } \\
\hline $\begin{array}{cc}\text { No. of } \\
\text { Sinks }\end{array}$ & $\begin{array}{c}\text { Covered Nodes } \\
(\%)\end{array}$ \\
\hline 1 & 100 \\
2 & 100 \\
3 & 100 \\
4 & 100 \\
6 & 100 \\
8 & 100 \\
24 & 100 \\
\hline
\end{tabular}

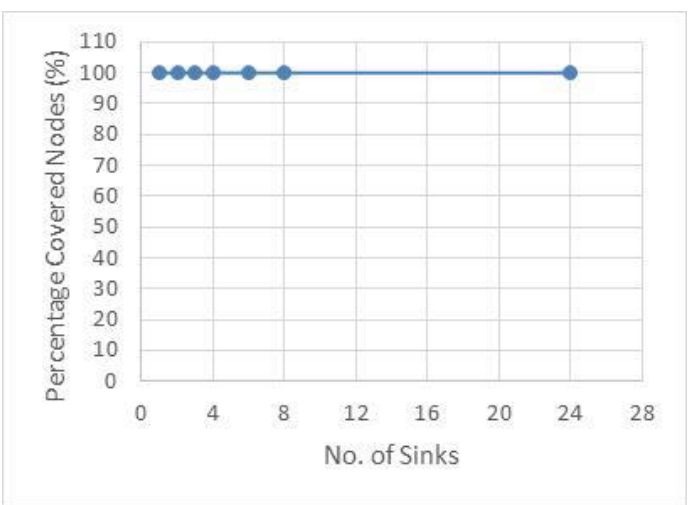

Figure 11. Percentage of covered nodes plotted against number of sinks (Total number of nodes $=140)$

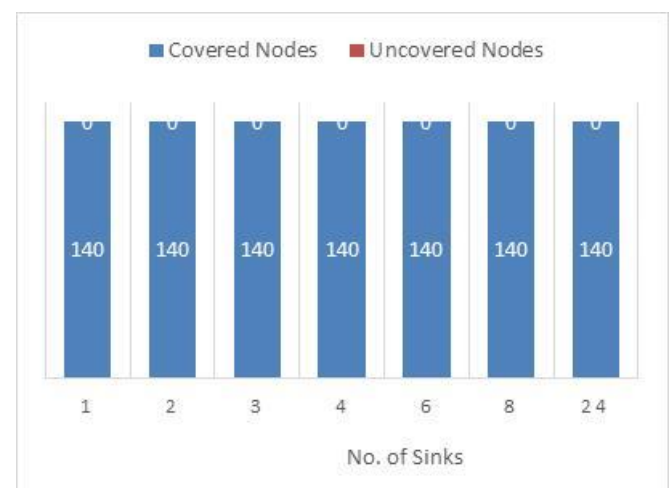

Figure 12. Actual number of nodes covered and uncovered against number of sinks. (Total no of nodes $=140$ )
Table 7. Actual Number of Nodes Covered and Uncovered with Various Number of Sinks Applied (Total number of nodes $=140$ )

\begin{tabular}{ccc}
\hline $\begin{array}{c}\text { No. of } \\
\text { Sinks }\end{array}$ & $\begin{array}{c}\text { Covered } \\
\text { Nodes }\end{array}$ & $\begin{array}{c}\text { Uncovered } \\
\text { Nodes }\end{array}$ \\
\hline 1 & 140 & 0 \\
2 & 140 & 0 \\
3 & 140 & 0 \\
4 & 140 & 0 \\
6 & 140 & 0 \\
8 & 140 & 0 \\
24 & 140 & 0 \\
\hline
\end{tabular}

As the number of sinks increase, the percentage number of nodes will be increase as well. It is because, more area can be covered which means, more nodes can be covered with multiple sinks applied to the system. The number of sinks applied to the system is increased in every run, starting with 1, 2, 3, 4, 6, 8 , and 24. Also, the system is being tested with 5 different simulation settings and the result is the same for all settings. The settings will not affect the coverage of nodes, but the number of sinks applied do have a considerable impact on performance.

From the results above, using $\operatorname{simB}$, it reflects a more realistic scenario for a city environment in contrast with $\operatorname{sim} A$. This happens because $\operatorname{simB}$ did not consider applying instantaneous shadowing effect to its system and vice versa. Hence, using simA, actual performance is more accurately reflected.

B. Percentage of Lost Packets

\section{$\operatorname{SimA}$}

Table 8. Number of Lost Packet During Transmission with Different Sets of Experiments and Different Number of Sinks Applied

\begin{tabular}{cccccc}
\hline No. of Sinks & Exp 0 & Exp 1 & Exp 2 & Exp 3 & Exp 4 \\
\hline 1 & 478 & 451 & 515 & 388 & 451 \\
2 & 466 & 468 & 482 & 384 & 400 \\
3 & 559 & 481 & 484 & 406 & 421 \\
4 & 524 & 469 & 504 & 390 & 414 \\
6 & 490 & 470 & 473 & 378 & 430 \\
8 & 478 & 463 & 464 & 382 & 389 \\
24 & 459 & 464 & 460 & 404 & 404 \\
\hline
\end{tabular}


With the same simulation (in the first part), the packet lost also can be identified. Unlike the first part, here, both of number of sinks and settings had made a greater impact to the system. The first setting (we called as Exp 0) is used the settings with the slowest data rate (SF12, BW125, CR4/8). The second setting (we called as Exp1) is also uses the slowest data rate as in Exp0, but different here is it use a random choice of three transmit frequencies.

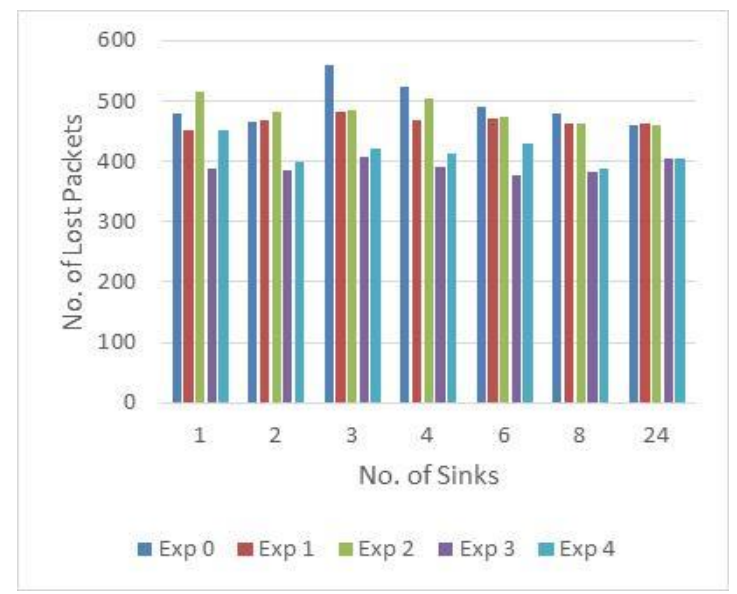

Figure 13. Average no. of lost packets vs. No. of sinks

\section{SimB}

Table 9. Number of Lost Packet During Transmission with Different Sets of Experiments and Different Number of Sinks Applied

\begin{tabular}{cccccc}
\hline No. of Sinks & Exp 0 & Exp 1 & Exp 2 & Exp 3 & Exp 4 \\
\hline 1 & 0 & 0 & 0 & 0 & 0 \\
2 & 65 & 71 & 70 & 81 & 65 \\
3 & 66 & 75 & 50 & 70 & 66 \\
4 & 25 & 54 & 53 & 68 & 49 \\
6 & 57 & 66 & 65 & 73 & 78 \\
8 & 43 & 72 & 70 & 73 & 94 \\
24 & 95 & 87 & 92 & 91 & 98 \\
\hline
\end{tabular}

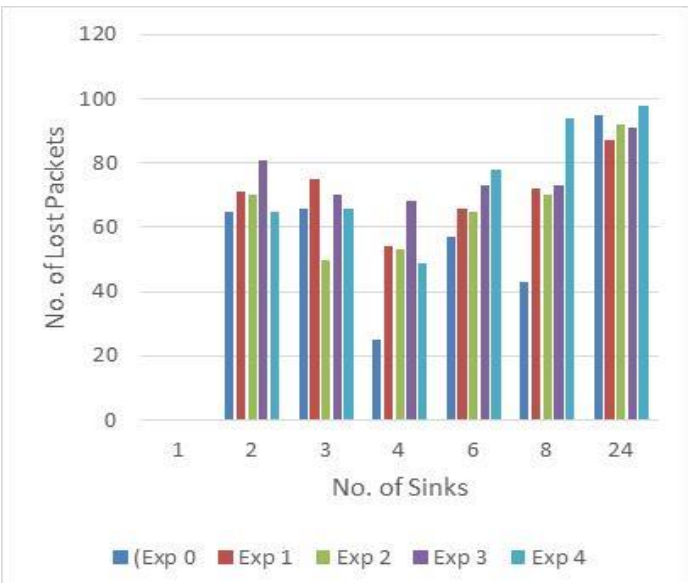

Figure 14. Average no. of lost packets vs. No. of sinks 
The third setting (we called as Exp2) is used the settings with the fastest data rate (SF6, BW500, CR4/5). The fourth setting (we called as Exp3) is used the settings as defined in LoRaWAN (SF12, BW125, CR4/5). Finally, the fifth setting (we called as Exp 4) is optimize the setting per node based on the distance to the gateway and the transmit power. Exp3 shows that, it has lower packet lost during transmission, following with $\operatorname{Exp} 4, \operatorname{Exp} 1, \operatorname{Exp} 2$, and Exp 0. The slowest data rate (which is Exp0) has greater packet lost compared to others. This is because, the Exp3 occupied with settings as defined in LoRaWAN, which promises greater area to cover and more successful transmission compared with others.

\section{CONCLUSION}

The work presented in this paper has reflected the effect of instantaneous and cumulative effect of shadowing on LoRa air interface for nodes distributed in a uniform grid-type formations representing typical utilities distribution in a city environment. The number of covered/uncovered nodes was studied together with number of sink nodes needed for practical network coverage. Furthermore, number of lost packets due to lost coverage was presented as a measure for with and without shadowing effect on the channel. Results indicate a considerable effect of shadowing on communication links resulting in lost packets and connectivity issues that need to be addressed carefully when designing the network.

\section{ACKNOWLEDGEMENTS} $362-0526$

This work is partially supported by International Islamic University Malaysia Grant No. RIGS16-

\section{REFERENCES}

[1] Nur Aishah Zainal, Mohamed Hadi Habaebi, Israth Jahan Chowdhury, and Md. Rafiqul Islam, "Cluttered traffic distribution in LoRa LPWAN", Indonesian Journal of Electrical Engineering and Computer Science, 10 (1). pp. 214-223, 2018.

[2] Pascal Thubert, Alexander Pelov ans Suresh Krishnan, "Low-Power Wide-Area Networks at the IETF," IEEE Commun. Stand. Mag., no. March, pp. 76-79, 2017.

[3] O. Georgiou and U. Raza, "Low Power Wide Area Network Analysis: Can LoRa Scale?," vol. 2337, no. c, pp. 1-4, 2016.

[4] S. Broome, "Microchip's LoRa ${ }^{\circledR}$ Wireless Module is World's First to Pass LoRa Alliance Certification", Microchip Technology Inc. Press Release 2015.

[5] A. Fallis, "Lora_FAQ," vol. 53, no. 9, pp. 1689-1699, 2013.

[6] Augustin, J. Yi, T. Clausen, and W. Townsley, "A Study of LoRa: Long Range \& Low Power Networks for the Internet of Things," Sensors, vol. 16, no. 9, p. 1466, 2016.

[7] Nicolas Sornin, "The LoRaWAN TM Specification Developments", LoRa-Alliance.org Technical Report. Available online on https://edoc.site/lorawan-specification-developments--pdf-free.html

[8] J. Petäjäjärvi, K. Mikhaylov, A. Roivainen, T. Hänninen, and M. Pettissalo, "On the coverage of LPWANs: Range evaluation and channel attenuation model for LoRa technology,” 2015 14th Int. Conf. ITS Telecommun. ITST 2015, pp. 55-59, 2016.

[9] M. C. Bor, U. Roedig, T. Voigt, and J. M. Alonso, "Do LoRa Low-Power Wide-Area Networks Scale?," Proc. 19th ACM Int. Conf. Model. Anal. Simul. Wirel. Mob. Syst., pp. 59-67, 2016.

[10] Nurul Afifah Binti Masadan, Mohamed Hadi Habaebi, Siti Hajar Yusoff, "LoRa LPWAN Propagation Channel Modelling in IIUM Campus", 2018 7th International Conference on Computer and Communication Engineering (ICCCE2018), KL - Malaysia, pp.: 14-19, 19/9/2018.

[11] Siti Hajar Yusoff Nurul Afifah Binti Masadan, Mohamed Hadi Habaebi, "Long Range Channel Characteristics Through Foliage", Bulletin of Electrical Engineering and Informatics 8 (2), pp.: 1-110, 2019.

[12] Asif M. Yousuf, Edward M. Rochester, Behnam Ousat and Majid Ghaderi, "Throughput, Coverage and Reliability of LoRa LPWAN for the Internet o Things", IEEE Sensors Journal, Early Access, pp.: 1-1, 2019, DOI:10.1109/JSEN2019.2897156.

[13] Santa, J., Sanchez-Iborra, R., Rodriguez-Rey, P., Bernal-Escobedo, L., Skarmeta, A.F., "LPWAN-Based Vehicular Monitoring Platform with a Generic IP Network Interface”, MDPI Sensors, 19(2), 264, pp.: 1-17, 2019.

[14] Polonelli, T., Brunelli, D., Marzocchi, A., Benini, L., "Slotted ALOHA on LoRaWAN-Design, Analysis, and Deployment", MDPI Sensors, 19(4), 838, 2019.

[15] K. A. Ahmad, J. D. Segaran, F. R. Hashim and M. T. Jusoh, "LoRa Propagation at 433 MH in Tropical Climate Environment”, J. undam Appl Sci, 9(3S), 384-394, 2017. 